\title{
Factores demográficos y clínicos que explican la progresión de la Enfermedad Renal Crónica en un programa de nefroprotección del departamento de Nariño, Colombia 2016-2018
}

Bertha Ligia Bernal Bastidas a.c ; Oscar Iván Quirós Gómez ${ }^{\text {b,c }}$

\begin{abstract}
a Médica general, Magíster en Epidemiología. ORCID: https://orcid.org/0000-0001-6640-6386 Autor corresponsal: belibeba232@ gmail.com. ; ${ }^{b}$ Doctor en Epidemiología y Bioestadística, Docente Facultad de Medicina c Universidad CES Medellín, Colombia. ORCID: https://orcid.org/0000-0002-5699-9912 DOI: https://doi.org/10.22517/25395203.24533
\end{abstract}

\section{Resumen}

Introducción: La Enfermedad Renal Crónica es una patología de gran impacto en salud pública a nivel mundial, su progresión está determinada por limitaciones en el control de sus factores de riesgo y pronóstico. Los programas de nefroprotección hacen seguimiento y control de los pacientes con la enfermedad, intentando llevarlos a cumplir las metas específicas de nefroprotección para limitar la progresión de la Enfermedad Renal Crónica.

Objetivo: Determinar los factores explicativos de la progresión de la Enfermedad Renal Crónica en pacientes atendidos en un programa de nefroprotección.

Materiales y métodos: Estudio analítico observacional de cohorte retrospectivo en 5872 pacientes con Enfermedad Renal Crónica en diferentes estadios, participantes de un programa de nefroprotección en el departamento de Nariño durante el período 2016-2018. La progresión de la ERC, correspondió a la disminución de la Tasa de Filtración Glomerular en $5 \mathrm{~mL} /$ $\mathrm{min} / 1.73 \mathrm{~m}^{2} \mathrm{o}$ más. La información se obtuvo de la base de datos del programa. Se construyó un modelo explicativo ajustado por variables demográficas y clínicas. Acorde al diseño se utilizó un modelo binomial con función de enlace logarítmica para estimar los coeficientes de regresión de las variables de interés. Las medidas de asociación fueron los Riesgos Relativos.

Resultados: el 72,2\% fueron mujeres, el 75,1\% tenían 60 años o más, el 12,4\% eran afrocolombinos, y el $85 \%$ mestizos, se presentó progresión el 49,2\% (IC 95\% 47,9-50,4) de los casos. Los factores que mejor explicaron la relación la progresión de ERC, fueron: ser hombre con un RR ajustado de 1,04 (IC95\%:1,00-1,15), y tener un estadio de la ERC, de 4, 5 y $3 a-b$, con RR ajustado 1,62 (IC1,36-1,94) y 1,41 (IC1,21-1,63) respectivamente.

Conclusiones: En la población nariñense de Colombia afiliada a un programa de nefroprotección, la progresión de la ERC fue del 49,2\%, siendo explicada de forma significativa por el sexo masculino, y los estadios avanzados de la enfermedad.

Palabras clave: Progresión de la enfermedad, diabetes mellitus tipo2, hipertensión, insuficiencia renal crónica.

\section{Demographic and clinical factors that explain the progression of Chronic Kidney Disease in a nephroprotection program in the department of Nariño, Colombia between 2016-2018.}

\begin{abstract}
Introduction: Chronic Kidney Disease is a pathology of great impact on public health worldwide, its progression is determined by limitations in the control of its risk factors and prognosis. Nephroprotection programs monitor, control, and limit the progression of this disease in the patients while trying to lead them to meet the specific program goals.
\end{abstract}

Objective: To determine the factors that boost the progression of Chronic Kidney Disease in patients treated in a nephroprotection program.

Materials and methods: This retrospective analytical observational cohort study was carried out in 5872 patients from a nephroprotection program with different stages of Chronic Kidney Disease, in the department of Nariño between 2016 and 2018. The progression of CKD corresponded to a decrease in the Glomerular Filtration Rate by $5 \mathrm{~mL} / \mathrm{min} / 1.73$ $\mathrm{m} 2$ or more; this information was obtained from the program's database. An explanatory model was built and adjusted taking into consideration demographic and clinical variables. According to the design, a binomial model with a logarithmic link function was used to estimate the regression coefficients of the variables of interest. The association measures were the Relative risks.

Results: In the final results it was found that $72.2 \%$ were women, $75.1 \%$ were 60 years old or older, $12.4 \%$ were afrocolombian, $85 \%$ were mixed race, and $49.2 \%$ presented progression $(95 \%$ CI $47,9-50.4)$ of the cases. The factors that best explained the relationship between CKD progression were: being a man with an adjusted RR of 1.04 (95\% CI: $1.00-$ $1.15)$, and having a CKD stage of 4,5 and $3 a-b$, with adjusted RR 1.62 (CI1.36-1.94) and 1.41 (CI1.21-1.63) respectively.

Conclusions: In the Nariño population of Colombia that is affiliated to a nephroprotection program the progression of CKD was $49.2 \%$ according to the male sex and the advanced stages of the condition.

Key words: disease progression, type 2 diabetes mellitus, hypertension, chronic kidney failure. 


\section{Introducción}

LaEnfermedadRenalCrónica(ERC) es una de las enfermedades de mayor impacto en la salud pública a nivel global, con una incidencia y prevalencia en aumento debido a factores como el proceso natural de nefroenvejecimiento que dificulta el diagnóstico de la ERC y la aparición de enfermedades como la Diabetes Mellitus (DM), Hipertensión Arterial (HTA) y obesidad que actuán como agentes etiológicos de la ERC (1).

La prevalencia estimada a nivel mundial de ERC es del 13,4\% $(11,7-15,1 \%)$, con alrededor de seis millones de pacientes en estadios terminales con necesidad de terapia de reemplazo renal. En algunos países, la prevalencia es aún mayor, como el caso de Estados Unidos y España con prevalencias de $14 \%$ y $20,6 \%$ en la población adulta (2), (3). En Colombia, según la cuenta de alto costo a junio de 2019, la prevalencia de la ERC era de $1,84 \%$ (4).

La progresión de la ERC es definida por el descenso de la Tasa de Filtración Glomerular (TFG) $>5 \mathrm{ml} / \mathrm{min} /$ año o > $10 \mathrm{ml} / \mathrm{min}$ en cinco años (5) y contribuye a la aparición de complicaciones agudas y crónicas que conllevan a aumento de la morbimortalidad en este grupo de pacientes (7). Sin embargo, la progresión es muy variable ya que depende de múltiples factores, entre ellos, la presencia de Hipertensión Arterial (HTA), Diabetes Mellitus (DM) y proteinuria (6).

En Colombia, desde el año 2008, se creó la Cuenta de Alto Costo (4) que obliga a las empresas prestadoras de servicios de salud reportar las enfermedades con costo elevado para el sistema, entre ellas la ERC. Posteriormente, en el año 2013, se creó el programa de nefroprotección que hace seguimiento de las enfermedades precursoras (HTA, DM) y a los ya diagnosticados, para realizar acciones oportunas de detección y tratamiento (10-11).

Diversas investigaciones muestran que hay factores demográficos y clínicos asociados a la progresión de la ERC (6), (9), por tanto, el objetivo de este estudio fue determinar los factores demográficos y clínicos que explican la progresión de pacientes con ERC en el contexto de un programa de nefroprotección.

\section{Materiales y métodos}

Estudio observacional de cohorte retrospectiva con pacientes de un programa de nefroprotección de una entidad promotora de salud que atiende principalmente el régimen subsidiado en el departamento de Nariño, Colombia. De una población de referencia de 6.707, se analizaron todos los pacientes que cumplieron con los criterios de inclusión, para un total de 5872 participantes.

Los criterios de inclusión fueron: 1) ser mayor de edad; 2) tener diagnóstico de ERC en cualquier estadio; 3) pertenecer al programa desde el año 2016, como mínimo. Se excluyeron pacientes gestantes y pacientes sin medición de la TFG. La primera medición de las variables de interés fue hecha en los primeros 5 meses de 2016, la segunda medición se realizó 2 años después, en la que 835 pacientes no se incluyeron por no contar con la medición de la TFG.
Se realizó el contraste de los valores de la TFG en donde una disminución delta de $5 \mathrm{ml} / \mathrm{min} / 1.73 \mathrm{~m}^{2}$ o más, indicaba progresión como variable dependiente (dicotómica si/no).

Las variables analizadas fueron de tipo demográfico y clínico, recolectadas de fuente secundaria (base de datos del programa de nefroprotección). La recolección de la información se hizo por personal médico del programa, previamente capacitado en los años 2016 y 2018.

Las variables independientes fueron:

a. Demográficas: sexo, edad (<de 60 años, > o igual a 60 años, parámetro tomado de cuenta de alto costo 2019), etnia, nivel de escolaridad, procedencia.

b. Clínicas:

Tabaquismo.

Exposición a biomasa.

Consumo de alcohol.

Actividad física.

Diagnóstico de HTA.

Compensación de tensión arterial (TA

no compensada si es menor de 60 años:

$>140 / 90 \mathrm{mmHg},>0$ igual a 60 años:

$>150 / 90 \mathrm{mmHg}$ ) (5).

Recibe IECA.

Recibe ARA II.

Diagnóstico de DM.

Clasificación de DM y compensación de DM

(se tomó de la base de datos, consideraron compensada si niveles de HbA1c menores de $7 \%$ si es menor de 60 años, y < o igual a $8 \%$ si es mayor o igual de 60 años).

Glucosa en ayunas (>126mg/dl, 110-126mg/dl, $<110 \mathrm{mg} / \mathrm{dl}$ ).

Glucosa posprandial (> o igual a $200 \mathrm{mg} / \mathrm{dl}$, $140-199,9 \mathrm{mg} / \mathrm{dl},<139,9 \mathrm{mg} / \mathrm{dl})(3)$.

Compensación de $\mathrm{HbA} 1 \mathrm{C}$ ( $<7 \%$ si es menor de

60 años, y < o igual a $8 \%$ si es mayor o igual de 60 años).

Diagnóstico de dislipidemias.

LDL ( $>$ o igual $100 \mathrm{mg} / \mathrm{dl},<100 \mathrm{mg} / \mathrm{dl}$ ).

HDL (si es hombre $<$ o igual a 35

descompensado, si es mujer <o igual a 40

descompensada) (3).

Colesterol total ( $>$ o igual a $200 \mathrm{mg} / \mathrm{dl}$

descompensado, <200mg/dl compensado) (3).

Triglicéridos ( $>$ o igual a $150 \mathrm{mg} / \mathrm{dl}$,

descompensado, <150mg/dl compensado) (3).

Recibe estatinas.

IMC (obesidad 1,2,3: IMC >0 igual a 30kg/ $\mathrm{m} 2$; sobrepeso $25 \mathrm{~kg} / \mathrm{m} 2-29.9 \mathrm{~kg} / \mathrm{m} 2$, bajo peso $<18,5 \mathrm{~kg} / \mathrm{m} 2$, peso normal $18,5 \mathrm{~kg} / \mathrm{m} 2-24,9 \mathrm{~kg} /$ m2) (3).

Perímetro abdominal (si es hombre $>0$ igual a $102 \mathrm{~cm}$ descompensado, si es mujer $<$ o igual a $88 \mathrm{~cm}$ descompensado) (3).

Adherencia al tratamiento (se tomó como variable de la base de datos, consideraron no adherencia si olvida tomar al menos una vez 
cualquiera de sus medicamentos de control de sus enfermedades).

Hb en metas (< o igual a $10 \mathrm{mg} / \mathrm{dl}$ baja, > a $10 \mathrm{mg} / \mathrm{dl}$ en metas) (5).

PTH en metas ( > a 110pg/ml alto, < o igual a $110 \mathrm{pg} / \mathrm{ml}$ en metas) (5).

Fósforo en metas $(>4,6 \mathrm{mg} / \mathrm{dl}, 2,7 \mathrm{mg} / \mathrm{dl}-4,6 \mathrm{mg} /$

$\mathrm{dl},<2,7 \mathrm{mg} / \mathrm{dl}$ en metas) (5).

Albumina $(<\mathrm{o}$ igual $4 \mathrm{~g} / \mathrm{dl}$ baja, $>4 \mathrm{~g} / \mathrm{dl}$ en metas) (9).

Potasio ( $>$ o igual a $5,5 \mathrm{meq} / 1$ elevado, $<$ 3,5meq/1 bajo, 3,5-5,49meq/1 en metas) (9). Creatinina sérica $(>1,5 \mathrm{mg} / \mathrm{dl}$ elevada, $0,5-$ $1,5 \mathrm{mg} / \mathrm{dl}$ normal) (9).

Cociente albumina/creatinina ( $>$ o igual a $300 \mathrm{mg} / \mathrm{gr}$ elevado, $31 \mathrm{mg} / \mathrm{dl}-299 \mathrm{mg} / \mathrm{gr}$ moderadamente elevado, $10 \mathrm{mg} / \mathrm{dl}-30 \mathrm{mg} / \mathrm{gr}$ levemente elevado, $<10 \mathrm{mg} / \mathrm{gr}$ normal) (9). Albuminuria $(>300 \mathrm{mg} / \mathrm{gr}$ incremento severo, $30-300 \mathrm{mg} / \mathrm{gr}$ moderado incremento, $<30 \mathrm{mg} / \mathrm{gr}$ normal o mínimo incremento) (9).

Etiología de la ERC.

Estadio de la ERC.

Todas las variables cuantitativas se recodificaron a cualitativas, siguiendo los indicadores de nefroprotección para pacientes con ERC, nacionales e internacionales (kdigo y CAC). Se utilizaron medidas de frecuencia absoluta y relativa para el análisis de las variables cualitativas. El análisis bivariado se realizó utilizando chi cuadrado. Para estimar la fuerza de asociación se usó el riesgo relativo (RR), con su respectivo intervalo de confianza de $95 \%$ (IC95\%) y se consideró significativo un valor $\mathrm{p}<0.005$.

Los factores independientes fueron determinados mediante modelos lineales generalizados de regresión binomial con enlace logarítmico, con fines explicativos, y para controlar posibles variables confusoras como: HTA, sexo, edad, estadio de la ERC, se incluyeron al modelo multivariado. Los paquetes estadísticos utilizados fueron SPSS 21.0 (licencia Universidad CES) y Epidat 3.1.

Para el presente trabajo se tuvo en cuenta la declaración de Helsinki para salvaguardar la confidencialidad de los participantes, se clasificó como investigación sin riesgo según el artículo 11 de la resolución 8430 de 1993 para Colombia, además, fue aceptado por el comité de ética institucional de la Universidad CES de Medellín y de la Institución Prestadora de Servicios de Salud.

\section{Resultados}

\section{Características demográficas y clínicas de los pacientes del estudio}

Se analizaron un total de 5872 pacientes, encontrando para los factores demográficos, que el $72,2 \%$ fueron mujeres, el $75,1 \%$ tenían 60 años o más. Con respecto a la etnia, el 12,4\% eran de raza negra siendo la mayoría mestizos con un total del $85 \%$. A nivel de escolarización, el $88,5 \%$ de los pacientes cursó estudios de básica primaria. En cuanto a la procedencia, el $47 \%$ de los participantes eran de la ciudad de Pasto.
Con respecto a las características clínicas se encontró que la exposición a biomasa fue del 15,4\% y más del $50 \%$ realizaba actividad física regular, con un 61,6\%. La mayoría de los pacientes tenían HTA, con un $95 \%$, el 78,5\% la tiene compensada, la mayoría de pacientes reciben ARA II $(71,5 \%)$, y solo el $17,6 \%$ recibe IECA. Más de la mitad de los pacientes tienen diagnóstico de dislipidemias $(65,2 \%)$, con un $67,9 \%$ de pacientes con colesterol total compensado, con la mitad de los pacientes con descompensación del colesterol LDL. La DM se presentó en el $25,1 \%$ de los casos, con un $17 \%$ de pacientes compensada, la glucemia en ayunas estuvo dentro de rangos normales en un $56,4 \%$, con respecto a la glucemia postprandial solo el 9,3\% la tenía compensada. Los pacientes tuvieron una $\mathrm{HbA} 1 \mathrm{C}$ descompensada en un 7,2\% de los casos. En cuanto a los triglicéridos, el $44,3 \%$ tienen este parámetro elevado. Los pacientes que reciben estatinas del total de la población son el 48,5\%. Con relación al IMC, el 68,4\% tienen sobrepeso $\mathrm{u}$ obesidad en cualquiera de sus grados, con un perímetro abdominal elevado en un $65 \%$.

Con respecto a la etiología del deterioro de la función renal, la mayoría de los pacientes tienen como factor causal la HTA o la DM con un $71,5 \%$ mientras que un $28,5 \%$ de los pacientes presentan otras causas. Con referencia al estadio de la ERC, más de la mitad (52,4\%) tienen un estadio de 3-5. En cuanto a los electrolitos como potasio y fósforo, la mayoría de los pacientes los tenía dentro de rangos normales, 74,4\%, y $47,1 \%$. La creatinina estaba elevada en un $52,3 \%$ de los casos. Finalmente, el 49,2\% tuvieron un deterioro de la función renal. En la Tabla1 se presentan las características demográficas y clínicas analizadas: 
Tabla1. Características demográficas y clínicas de los pacientes del estudio.

\begin{tabular}{|c|c|c|c|}
\hline \multicolumn{4}{|c|}{ FACTORES DEMOGRÁFICOS } \\
\hline & $n(\%)$ & & $n(\%)$ \\
\hline $\begin{array}{l}\text { Sexo } \\
\text { Hombre } \\
\text { Mujer }\end{array}$ & $\begin{array}{l}1634(27.8) \\
4238(72.2)\end{array}$ & $\begin{array}{l}\text { Tabaquismo } \\
\mathrm{Si} \\
\mathrm{No}\end{array}$ & $\begin{array}{c}192(3.3) \\
5680(96.7)\end{array}$ \\
\hline $\begin{array}{l}\text { Edad } \\
>=60 \text { años } \\
<60 \text { años }\end{array}$ & $\begin{array}{l}4409(75.1) \\
1637(24.9)\end{array}$ & $\begin{array}{l}\text { Exposición a } \\
\text { biomasa } \\
\mathrm{Si} \\
\text { No }\end{array}$ & $\begin{array}{c}904(15.4) \\
4968(84.6)\end{array}$ \\
\hline $\begin{array}{l}\text { Etnia } \\
\text { Negro } \\
\text { Gitano-Indígena } \\
\text { Mestizo }\end{array}$ & $\begin{array}{c}731(12.5) \\
150(2.5) \\
4991(85)\end{array}$ & $\begin{array}{l}\text { Consumo de alcohol } \\
\mathrm{Si} \\
\text { No }\end{array}$ & $\begin{array}{c}247(4.2) \\
5625(95.8)\end{array}$ \\
\hline $\begin{array}{l}\text { Escolaridad } \\
\text { Primaria inco-comp } \\
\text { bachillerato inco-comp } \\
\text { Técnico-Universitario }\end{array}$ & $\begin{array}{c}5197(88.5) \\
597(10.2) \\
78(1.3)\end{array}$ & $\begin{array}{l}\text { Realiza actividad } \\
\text { física } \\
\mathrm{Si} \\
\text { No }\end{array}$ & $\begin{array}{l}3618 \text { (61.6) } \\
2254(38.4)\end{array}$ \\
\hline $\begin{array}{l}\text { Procedencia } \\
\text { Pasto-Ipiales } \\
\text { Tumaco-Túquerres } \\
\text { Otros }\end{array}$ & $\begin{array}{l}2760(47) \\
2384(40.6) \\
728(12.4)\end{array}$ & & \\
\hline \multicolumn{4}{|l|}{ FACTORES CLÍNICOS } \\
\hline $\begin{array}{l}\text { Diagnostico HTA } \\
\mathrm{Si} \\
\text { No }\end{array}$ & $\begin{array}{c}5576(95) \\
296(5)\end{array}$ & $\begin{array}{l}\text { Diagnostico DM } \\
\mathrm{Si} \\
\text { No }\end{array}$ & $\begin{array}{l}1476(25.1) \\
4396(74.9)\end{array}$ \\
\hline $\begin{array}{l}\text { Compensación de } \\
\text { HTA } \\
\mathrm{Si} \\
\text { No }\end{array}$ & $\begin{array}{l}4609(78.5) \\
1263(21.5)\end{array}$ & $\begin{array}{l}\text { Clasificación de DM } \\
\text { DM1 } \\
\text { DM2 } \\
\text { Prediabetes } \\
\text { Sin DM }\end{array}$ & $\begin{array}{c}6(0.1) \\
1470(25) \\
1243(21.2) \\
3153(53.7)\end{array}$ \\
\hline $\begin{array}{l}\text { Recibe IECA } \\
\mathrm{Si} \\
\text { No }\end{array}$ & $\begin{array}{l}1031(17.6) \\
4841(82.4)\end{array}$ & $\begin{array}{l}\text { Compensación de } \\
\text { DM } \\
\text { No compensada } \\
\text { Compensada } \\
\text { No tiene DM }\end{array}$ & $\begin{array}{c}470(8) \\
1006(17.1) \\
4396(74.9)\end{array}$ \\
\hline $\begin{array}{l}\text { Recibe ARA II } \\
\mathrm{Si} \\
\text { No }\end{array}$ & $\begin{array}{l}4196(71.5) \\
1676(28.5)\end{array}$ & $\begin{array}{l}\text { Glucosa en ayunas } \\
>126 \\
110-126 \\
<110\end{array}$ & $\begin{array}{c}764(13) \\
1795(30.6) \\
3313(56.4)\end{array}$ \\
\hline $\begin{array}{l}\text { Diagnostico } \\
\text { dislipidemias } \\
\mathrm{Si}\end{array}$ & $3829(65.2)$ & $\begin{array}{l}\text { Glucosa } \\
\text { postprandial } \\
>=200\end{array}$ & $437(7.4)$ \\
\hline No & $2043(34.8)$ & $\begin{array}{l}140-199,9 \\
<139,9 \\
\text { No tiene DM }\end{array}$ & $\begin{array}{c}490(8.3) \\
549(9.3) \\
4396(74.9)\end{array}$ \\
\hline $\begin{array}{l}\text { Colesterol total } \\
>=200 \\
<200\end{array}$ & $\begin{array}{l}1887(32.1) \\
3985(67.9)\end{array}$ & $\begin{array}{l}\text { HbA1c } \\
\text { Descompensada } \\
\text { Compensada } \\
\text { No tiene DM }\end{array}$ & $\begin{array}{c}421(7.2) \\
1860(31.7) \\
3591(61.2)\end{array}$ \\
\hline $\begin{array}{l}\text { Colesterol LDL } \\
>=100 \\
<100\end{array}$ & $\begin{array}{l}2982(50.8) \\
2890(49.2)\end{array}$ & $\begin{array}{l}\text { Adherencia al } \\
\text { tratamiento } \\
\mathrm{Si} \\
\text { No }\end{array}$ & $\begin{array}{l}4247(72.3) \\
1625(27.7)\end{array}$ \\
\hline $\begin{array}{l}\text { Colesterol HDL } \\
\text { Descompensado } \\
\text { Compensado }\end{array}$ & $\begin{array}{l}1067(18.2) \\
4805(81.8)\end{array}$ & $\begin{array}{l}\text { Parathormona } \\
>110 \\
<=110 \\
\text { Perdidos }\end{array}$ & $\begin{array}{c}170(2.9) \\
2690(45.8) \\
3012(51.3)\end{array}$ \\
\hline $\begin{array}{l}\text { Triglicéridos } \\
>=150 \\
<150\end{array}$ & $\begin{array}{l}2601(44.3) \\
3271(55.7)\end{array}$ & $\begin{array}{l}\text { Albumina } \\
<=4 \\
>4 \\
\text { No aplica }\end{array}$ & $\begin{array}{c}353(6) \\
2610(44.4) \\
2909(49.5)\end{array}$ \\
\hline $\begin{array}{l}\text { Recibe estatinas } \\
\mathrm{Si} \\
\text { No }\end{array}$ & $\begin{array}{l}2846(48.5) \\
3026(51.5)\end{array}$ & $\begin{array}{l}\text { Potasio } \\
>=5,5 \\
3,5-5,49 \\
<3,5 \\
\text { Perdidos }\end{array}$ & $\begin{array}{c}284(4.8) \\
4371(74.4) \\
129(2.2) \\
1088(18.5)\end{array}$ \\
\hline $\begin{array}{l}\text { IMC } \\
\text { Obesidad I-I-III } \\
\text { Sobrepeso } \\
\text { Bajo peso } \\
\text { Peso normal }\end{array}$ & $\begin{array}{c}1633(27.8) \\
2382(40.6) \\
86(1.5) \\
1771(30.2)\end{array}$ & $\begin{array}{l}\text { Fosforo } \\
<2,7 \\
2,7-4,6 \\
>4,6 \\
\text { No aplica }\end{array}$ & $\begin{array}{c}61(1) \\
2765(47.1) \\
213(3.6) \\
2833(48.2)\end{array}$ \\
\hline $\begin{array}{l}\text { Perímetro abdominal } \\
\text { Elevado } \\
\text { Normal }\end{array}$ & $\begin{array}{l}3816(65) \\
2056(35)\end{array}$ & $\begin{array}{l}\text { Hemoglobina } \\
<=10 \\
>10 \\
\text { Perdidos }\end{array}$ & $\begin{array}{c}43(0.7) \\
4766(81.2) \\
1055(17.7)\end{array}$ \\
\hline $\begin{array}{l}\text { Etiología de la ERC } \\
\text { HTA o DM } \\
\text { Otras }\end{array}$ & $\begin{array}{l}4264(71.5) \\
1697(28.5)\end{array}$ & $\begin{array}{l}\text { Creatinina } \\
>1,5 \\
0,5-1,5\end{array}$ & $\begin{array}{l}3070(52.3) \\
2802(47.7)\end{array}$ \\
\hline $\begin{array}{l}\text { Estadio ERC } \\
\text { Estadio 5-4 } \\
\text { Estadio 3a-b } \\
\text { Estadio 2-1 }\end{array}$ & $\begin{array}{c}578(9.8) \\
2500(42.6) \\
2794(47.6)\end{array}$ & $\begin{array}{l}\text { Cociente } \\
\text { albumina/creatinina } \\
<10 \\
10-30 \\
31-299 \\
>=300\end{array}$ & $\begin{array}{c}1365(23.2) \\
801(13.6) \\
544(9.3) \\
3162(53.8)\end{array}$ \\
\hline $\begin{array}{l}\text { Deterioro de la función } \\
\text { renal } \\
\text { Si } \\
\text { No }\end{array}$ & $\begin{array}{l}2887(49.2) \\
2985(50.8)\end{array}$ & $\begin{array}{l}\text { Albuminuria } \\
>300 \\
30-300 \\
<30\end{array}$ & $\begin{array}{c}20(0.3) \\
854(14.5) \\
4998(85.1)\end{array}$ \\
\hline
\end{tabular}

HTA: hipertensión arterial, DM: diabetes mellitus, IECA: inhibidor de la enzima convertidora de angiotensina, ARA II: antagonistas competitivos de los receptores de angiotensina II, LDL: lipoproteínas de baja densidad, HDL: lipoproteínas de alta densidad, IMC: índice de masa corporal, ERC: enfermedad renal crónica.

\section{Características clínicas de los pacientes de estudio y su relación con la progresión de la ERC.}

De los pacientes pertenecientes al programa de nefroprotección entre 2016 y 2018, se encontró que el 49,2\% presentaban progresión de la ERC, correspondiente a la disminución de la TFG $5 \mathrm{~mL} / \mathrm{min} / 1.73 \mathrm{~m}^{2}$ en o más. La progresión de la ERC es más alta al superar los 60 años con un riesgo relativo de 1,07 veces de presentar deterioro de la TFG, al comparar con pacientes de menos de 60 años RR: 1,07 (IC95\% 1,00-1,14) valor $\mathrm{p}<0,03$.

Por su parte, la escolaridad, la exposición a biomasa y no realizar actividad física fueron variables que no tuvieron un valor significativo en la progresión de la ERC. Con relación a la procedencia, los datos indican que la mayoría de los pacientes participantes en el programa de nefroprotección son de la ciudad de Pasto y de Ipiales, con un riesgo relativo de 1,26 veces de presentar progresión de la ERC en comparación con pacientes procedentes de ciudades más pequeñas $O$ municipios alejados RR: 1,26 (IC95\% 08- 1,17) valor $\mathrm{p}<0,05$.

De las variables clínicas se encontró explicación cruda con la compensación de la TA, glucosa posprandial, colesterol LDL, IMC, fósforo, potasio, creatinina, cociente albuminuria/ creatinuria. La no adherencia al tratamiento tiene un riesgo relativo 1,18 veces de presentar progresión de la ERC, con respecto a un paciente adherente RR: 1,18 (IC95\% 1,12 $1,24)$, otra variable a analizar es el estadio de la ERC, de 3 a 5 , donde hay un riesgo relativo de 1,23 y 1,21 veces de presentar progresión de la enfermedad que pacientes en estadios 1-2. La variable etiología de la ERC, presenta datos significativos, con RR: 1,08 (IC95\%:1,05-1,11), de manera específica tener diagnóstico de HTA y/o DM, tiene un riesgo relativo de 1,08 veces de presentar deterioro crónico de la TFG, otras variables se presentan en la Tabla 2: 
Tabla 2. Características clínicas de los pacientes de estudio y su relación con la progresión de la ERC.

\begin{tabular}{|c|c|c|c|c|}
\hline \multirow{3}{*}{$\begin{array}{c}\text { FACTORES } \\
\text { DEMOGRÁFICOS }\end{array}$} & \multicolumn{2}{|c|}{ Deterioro de la Función renal } & \multirow[b]{3}{*}{ Valor P } & \multirow[b]{3}{*}{ RRc (IC95\%) } \\
\hline & \multirow{2}{*}{$\begin{array}{c}\text { SI } \\
n(\%)\end{array}$} & NO & & \\
\hline & & n (\%) & & \\
\hline Sexo & & & 0.743 & \\
\hline Hombre & $809(49.5)$ & $825(50.5)$ & & $1.01(0.95-1.07)$ \\
\hline Mujer & $2078(49)$ & $2160(51)$ & & Ref. \\
\hline Edad & & & 0.033 & \\
\hline$>=60$ años & $2203(50)$ & $2206(50)$ & & $1.07(1.00-1.14)$ \\
\hline$<60$ años & $684(46.8)$ & $779(53.2)$ & & Ref. \\
\hline Etnia & & & $<0.001$ & \\
\hline Negro & $250(34.2)$ & $481(65.8)$ & & $0.84(0.81-0.88)$ \\
\hline Indígena-gitano & $1(50)$ & $1(50)$ & & $0.99(0.92-1.08)$ \\
\hline Mestizo & $75(50.7)$ & $73(49.3)$ & & Ref. \\
\hline Escolaridad & & & 0.016 & \\
\hline Primaria incompleta & $2523(48.5)$ & $2674(51.5)$ & & $0.79(0.66-0.94)$ \\
\hline $\begin{array}{l}\text { Bachillerato comp- } \\
\text { incom }\end{array}$ & $317(53.1)$ & $280(46.9)$ & & $0.81(0.66-0.97)$ \\
\hline Tec- universitario & $47(60.3)$ & $31(39.7)$ & & Ref. \\
\hline Procedencia & & & $<0.001$ & \\
\hline Pasto & $1686(61.2)$ & $1071(38.8)$ & & $1.26(1.08-1.17)$ \\
\hline $\begin{array}{l}\text { Tumaco-Tuquerres- } \\
\text { Ipiales }\end{array}$ & $840(35.2)$ & $1543(64.8)$ & & $0.87(0.83-0.91)$ \\
\hline Otros & $361(49.3)$ & $371(50.7)$ & & Ref. \\
\hline Tabaquismo activo & & & 0.814 & \\
\hline Sí & $96(50)$ & $96(50)$ & & $1.02(0.88-1.18)$ \\
\hline No & 2791 (49.1) & $2889(50.9)$ & & Ref. \\
\hline $\begin{array}{l}\text { Exposición a } \\
\text { biomasa }\end{array}$ & & & $<0.001$ & \\
\hline Sí & 336 (37.2) & $568(62.8)$ & & $0.72(0.66-0.79)$ \\
\hline No & $2551(51.3)$ & 2417 (48.7) & & Ref. \\
\hline $\begin{array}{l}\text { Consumo de } \\
\text { alcohol }\end{array}$ & & & 0.751 & \\
\hline Sí & $119(48.2)$ & $128(51.8)$ & & $0.98(0.86-1.11)$ \\
\hline No & 2768 (49.2) & $2857(50.8)$ & & Ref. \\
\hline $\begin{array}{l}\text { Realiza actividad } \\
\text { física }\end{array}$ & & & $<0.001$ & \\
\hline No & $947(42)$ & $1307(58)$ & & $0.78(0.74-0.83)$ \\
\hline Sí & $1940(53.6)$ & $1678(46.4)$ & & Ref. \\
\hline
\end{tabular}


Deterioro de la Función renal

\begin{tabular}{|c|c|c|c|c|}
\hline \multirow{2}{*}{$\begin{array}{l}\text { FAČTORES } \\
\text { CLINICOS }\end{array}$} & \multicolumn{2}{|r|}{ NO } & \multirow[b]{2}{*}{ Valor $\mathbf{P}$} & \multirow[b]{2}{*}{ RRc (IC95\%) } \\
\hline & $\mathbf{N}(\%)$ & $\mathbf{N}(\%)$ & & \\
\hline Dx de HTA & & & 0.861 & \\
\hline $\mathrm{Si}$ & $2740(49.1)$ & $2836(50.9)$ & & $1.00(0.88-1.11)$ \\
\hline No & $147(49.7)$ & $149(50.3)$ & & \\
\hline $\begin{array}{l}\text { Compensación de la } \\
\text { TA }\end{array}$ & & & $<0.001$ & \\
\hline No compensada & $524(41.5)$ & 739 (58.5) & & $0.81(0.75-0.87)$ \\
\hline Compensada & $2362(51.3)$ & $2246(48.7)$ & & Ref. \\
\hline Recibe ARA II & & & 0.729 & \\
\hline Sí & $2057(49)$ & $2139(51)$ & & $0.99(0.94-1.05)$ \\
\hline No & $830(49.5)$ & $846(50.5)$ & & Ref. \\
\hline Recibe IECA & & & 0.269 & \\
\hline $\mathrm{Si}$ & $523(50.7)$ & 508 (49.3) & & $1.04(0.97-1.11)$ \\
\hline No & $2364(48.8)$ & 2477 (51.2) & & Ref. \\
\hline Diagnóstico de DM & & & 0.297 & \\
\hline Sí & $743(50.3)$ & 733 (49.7) & & $1.03(0.97-1.09)$ \\
\hline No & $2144(48.8)$ & 2252 (51.2) & & Ref. \\
\hline Clasificación de DM & & & 0.067 & \\
\hline DM1 & $4(66.7)$ & $2(33.3)$ & & $1.16(0.81-1.71)$ \\
\hline DM2 & $739(50.3)$ & $731(49.7)$ & & $0.99(0.95-1.03)$ \\
\hline Glicemia alterada & $641(51.6)$ & $602(48.4)$ & & Ref. \\
\hline No tiene DM & $1503(47.7)$ & $1650(52.3)$ & & \\
\hline Compensación DM & & & 0.461 & \\
\hline No compensada & $230(48.9)$ & $240(51.1)$ & & $0.96(0.86-1.07)$ \\
\hline Compensada & $513(51)$ & $493(49)$ & & Ref. \\
\hline No tiene DM & $2144(48.8)$ & $2252(51.2)$ & & \\
\hline Glucosa en ayunas & & & 0.076 & \\
\hline Mayor a $126 \mathrm{mg} / \mathrm{dl}$ & $353(46.2)$ & $411(53.8)$ & & $0.95(0.87-1.03)$ \\
\hline $110-126 \mathrm{mg} / \mathrm{dl}$ & $915(51)$ & $880(49)$ & & $1.05(0.99-1.11)$ \\
\hline Menor a $110 \mathrm{mg} / \mathrm{dl}$ & $1619(48.9)$ & 1694 (51.1) & & Ref. \\
\hline $\begin{array}{l}\text { Glucosa } \\
\text { postprandial }\end{array}$ & & & 0.023 & \\
\hline $\begin{array}{l}\text { Mayor o igual a } \\
200 \mathrm{mg} / \mathrm{dl}\end{array}$ & $201(46)$ & $236(54)$ & & $0.92(0.86-0.98)$ \\
\hline $140 \mathrm{mg} / \mathrm{dl}-199,9 \mathrm{mg} / \mathrm{dl}$ & $242(49.4)$ & $248(50.6)$ & & $0.95(0.89-1.00)$ \\
\hline Menor a $140 \mathrm{mg} / \mathrm{dl}$ & $300(54.6)$ & $249(45.4)$ & & Ref. \\
\hline No tiene DM & $2144(48.8)$ & $2254(51.2)$ & & \\
\hline
\end{tabular}




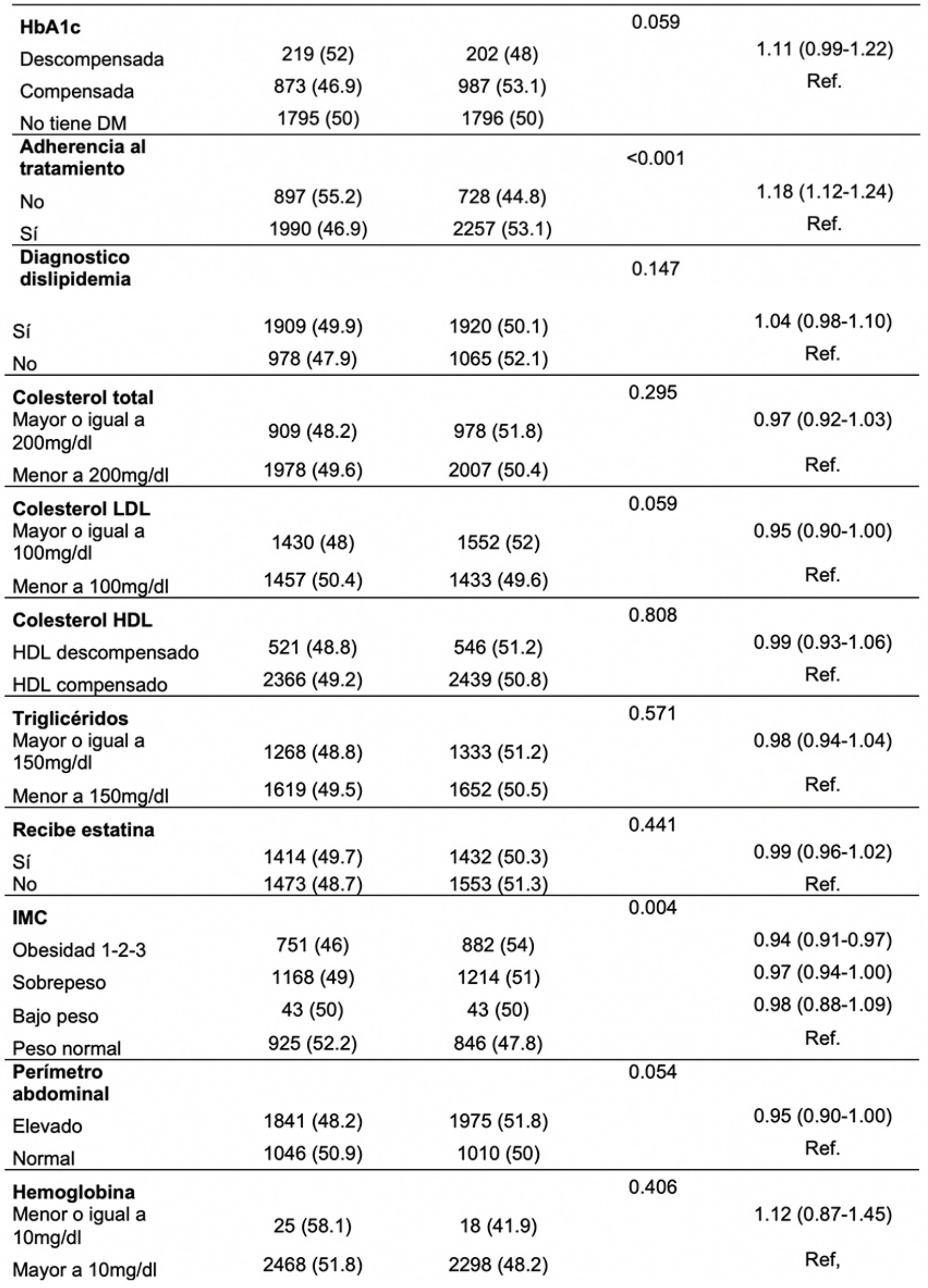




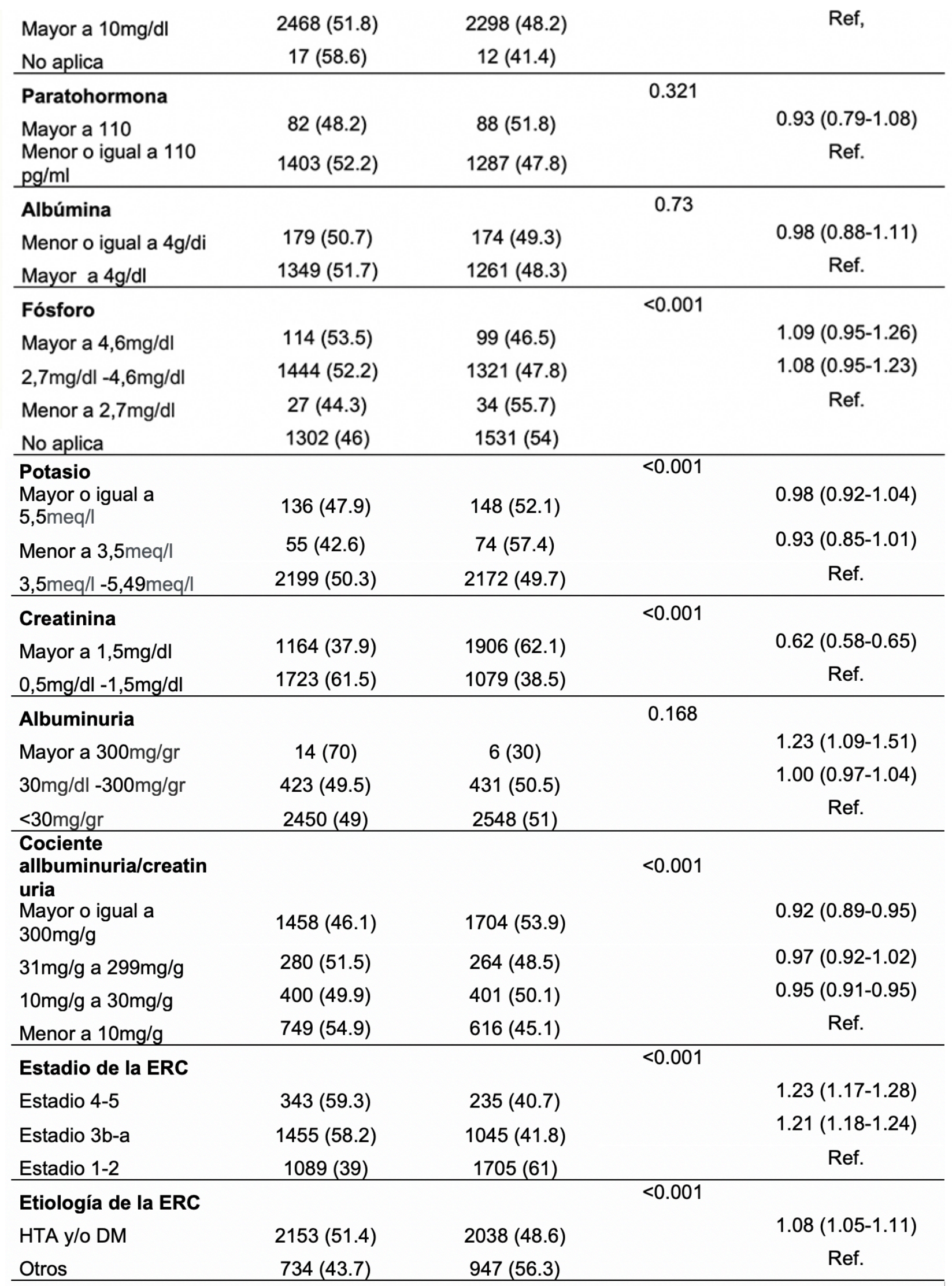

HTA: hipertensión arterial, DM: diabetes mellitus, IECA: inhibidor de la enzima convertidora de angiotensina, ARA II: antagonistas competitivos de los receptores de angiotensina II, LDL: lipoproteínas de baja densidad, HDL: lipoproteínas de alta densidad, IMC: índice de masa corporal, ERC: enfermedad renal crónica, TFG: tasa de filtración glomerular, HbAlc: hemoglobina glicosilada, TA: tensión arterial. 
Por otro lado, se puede evidenciar que algunas variables en una comparación unicausal presentan valores protectores en su RR, como el caso de la compensación de TA: RR: 0,81 IC95\%0,75-0,87, o el caso del valor de la glucosa posprandial > o igual 200mg/ dl, RR 0,92 IC95\%0,86-0,98.

En síntesis, se encontró que los factores que mejor explican la relación entre la progresión de la ERC y las distintas variables demográficas y clínicas fueron: ser hombre con un RR crudo de 1,01 IC95\% (0,95-1,07), con un RR ajustado de 1,04 (IC95\%:1,00$1,15)$, y tener un estadio de la ERC, de 4,5 y $3 \mathrm{a}-\mathrm{b}$, con RR ajustado $1,62(\mathrm{IC} 1,36-1,94)$ y $1,41(\mathrm{IC} 1,21-1,63)$ respectivamente. En la Tabla 3 se presentan los factores más influyentes en la progresión de la ERC:

Tabla 3. Factores demográficos y clínicos que mejor explican la progresión de la ERC, en un programa de nefroprotección 2016-2018.

\begin{tabular}{|c|c|c|c|c|}
\hline \multirow{3}{*}{$\begin{array}{l}\text { FACTORES } \\
\text { DEMOGRÁFICOS Y } \\
\text { CLÍNICOS }\end{array}$} & \multicolumn{2}{|c|}{ Deterioro de la función renal } & \multirow[b]{3}{*}{ RRc (IC95\%) } & \multirow[b]{3}{*}{ RRa (IC95\%) } \\
\hline & $\mathrm{SI}$ & NO & & \\
\hline & $\mathrm{N}(\%)$ & $N(\%)$ & & \\
\hline \multicolumn{5}{|l|}{ Sexo } \\
\hline Hombre & $809(49.5)$ & $825(50.5)$ & $1.01(0.95-1.07)$ & $1.04(1.00-1.15)$ \\
\hline Mujer & $2078(49)$ & $2160(51)$ & Ref. & Ref. \\
\hline \multicolumn{5}{|l|}{ Edad } \\
\hline$>=60$ años & $2230(50)$ & $2206(50)$ & $1.07(1.00-1.14)$ & $1.01(0.83-1.24)$ \\
\hline$<60$ años & $684(46.8)$ & $779(53.2)$ & Ref. & Ref. \\
\hline \multicolumn{5}{|l|}{ Etnia } \\
\hline Negro & $250(34.2)$ & $481(65.8)$ & $0.84(0.81-0.88)$ & $1.00(0.83-1.18)$ \\
\hline Indígena-gitano & $1(50)$ & $1(50)$ & $0.99(0.92-1.08)$ & $1.11(0.80-1.54)$ \\
\hline Mestizo & $75(50.7)$ & $73(49.3)$ & Ref. & Ref. \\
\hline \multicolumn{5}{|l|}{ Dx de HTA } \\
\hline $\mathrm{Si}$ & $2740(49.1)$ & $2836(50.9)$ & $0.99(0.88-1.11)$ & $0.97(0.83-1.14)$ \\
\hline No & $147(49.7)$ & $149(50.3)$ & Ref. & Ref. \\
\hline \multicolumn{5}{|l|}{ Compensación de TA } \\
\hline No compensada & $524(41.5)$ & $739(58.5)$ & $0.81(0.75-0.87)$ & $1.00(0.89-1.12)$ \\
\hline Compensada & $2362(51.3)$ & $2246(48.7)$ & Ref. & Ref. \\
\hline \multicolumn{5}{|l|}{ HbA1c } \\
\hline Descompensada & $219(52)$ & $202(48)$ & $1.11(1.00-1.23)$ & $1.00(0.90-1.13)$ \\
\hline Compensada & $873(46.9)$ & $987(53.1)$ & Ref. & Ref. \\
\hline \multicolumn{5}{|l|}{ No tiene DM } \\
\hline \multicolumn{5}{|l|}{ IMC } \\
\hline Obesidad 1-2-3 & $751(46)$ & $882(54)$ & $0.91(0.85-0.97)$ & $1.01(0.89-1.14)$ \\
\hline Sobrepeso & $1168(49)$ & $1214(51)$ & $0.94(0.88-1.00)$ & $1.04(0.94-1.16)$ \\
\hline Bajo peso & $43(50)$ & $43(50)$ & $0.97(0.76-1.23)$ & $0.92(0.66-1.30)$ \\
\hline Peso normal & $925(52.2)$ & $846(47.8)$ & Ref. & Ref. \\
\hline $\begin{array}{l}\text { Colesterol LDL } \\
\text { Mayor o igual a } \\
100 \mathrm{mg} / \mathrm{dl}\end{array}$ & $1430(48)$ & $1552(52)$ & $0.95(0.90-1.00)$ & $1.03(0.94-1.25)$ \\
\hline Menor a $100 \mathrm{mg} / \mathrm{dl}$ & $1457(50.4)$ & $1433(49.6)$ & Ref. & Ref. \\
\hline \multicolumn{5}{|l|}{ Triglicéridos } \\
\hline $\begin{array}{l}\text { Mayor o igual a } \\
150 \mathrm{mg} / \mathrm{dl}\end{array}$ & $1268(48.8)$ & $1333(51.2)$ & $0.98(0.94-1.04)$ & $0.94(0.86-1.03)$ \\
\hline Menor a $150 \mathrm{mg} / \mathrm{dl}$ & 1619 (49.5) & $1652(50.5)$ & Ref. & Ref. \\
\hline
\end{tabular}




\begin{tabular}{|c|c|c|c|c|}
\hline \multicolumn{5}{|l|}{$\begin{array}{l}\text { Adherencia al } \\
\text { tratamiento }\end{array}$} \\
\hline No & $897(55.2)$ & $728(44.8)$ & $1.18(1.12-1.24)$ & $1.03(0.94-1.12)$ \\
\hline Sí & $1990(46.9)$ & $2257(53.1)$ & Ref. & Ref. \\
\hline \multicolumn{5}{|l|}{ Albuminuria } \\
\hline Mayor a $300 \mathrm{mg} / \mathrm{gr}$ & $14(70)$ & $6(30)$ & $1.23(1.09-1.51)$ & $0.93(0.69-1.25)$ \\
\hline $30 \mathrm{mg} / \mathrm{dl}-300 \mathrm{mg} / \mathrm{gr}$ & $423(49.5)$ & $431(50.5)$ & $1.00(0.97-1.04)$ & $0.94(0.85-1.04)$ \\
\hline$<30 \mathrm{mg} / \mathrm{gr}$ & $2450(49)$ & $2548(51)$ & Ref. & Ref. \\
\hline \multicolumn{5}{|l|}{ Fósforo } \\
\hline Mayor a 4,6mg/dl & $114(53.5)$ & $99(46.5)$ & $1.09(0.95-1.26)$ & $1.15(0.76-1.73)$ \\
\hline $2,7 \mathrm{mg} / \mathrm{dl}-4,6 \mathrm{mg} / \mathrm{dl}$ & $1444(52.2)$ & $1321(47.8)$ & $1.08(0.95-1.23)$ & $1.21(0.83-1.78)$ \\
\hline Menor a 2,7mg/dl & $27(44.3)$ & $34(55.7)$ & Ref. & Ref. \\
\hline \multicolumn{5}{|l|}{ Estadio de ERC } \\
\hline Estadio 4-5 & $343(59.3)$ & $235(40.7)$ & $1.34(1.24-1.45)$ & $1.62(1.36-1.94)$ \\
\hline Estadio3b-a & $1455(58.2)$ & $1045(41.8)$ & $1.12(1.14-1.27)$ & $1.41(1.21-1.63)$ \\
\hline Estadio 2-1 & $1039(39)$ & $1705(61)$ & Ref. & Ref. \\
\hline
\end{tabular}

RRc: riesgo relativo crudo, RRa: riesgo relativo ajustado, DM: diabetes mellitus, Ref.: valor de referencia, IMC: índice de masa corporal, ERC: enfermedad renal crónica, RRc: riesgo relativo crudo, RRa: riesgo relativo ajustado, HTA: hipertensión arterial, TA: tensión arterial, DM: diabetes mellitus, mg/dl: miligramos por decilitro, HbA1C: hemoglobina glicosilada, IMC: índice de masa corporal, ERC: enfermedad renal crónica.

\section{Discusión}

Este estudio aporta información epidemiológica importante sobre la progresión de la ERC en cuanto a su asociación explicativa con factores demográficos y clínicos en una población adulta atendida en el programa de nefroprotección de la IPS CooemssanarPasto.

Con respecto a los factores demográficos, en el presente estudio la mayoría de pacientes con ERC fueron mujeres, en relación con la presentación de la enfermedad desde hace varios años en Colombia, $(4,8,12)$, debido posiblemente a que las mujeres acuden con mayor frecuencia a los servicios de salud que los hombres, además se debe tener en cuenta que la pirámide poblacional a partir de los 40 años es mayor en mujeres $(4,13)$; pero se encontró similitudes con otros estudios en cuanto a reconocer al sexo masculino como un factor explicativo a la progresión de la ERC, como en un estudio de cohorte prospectiva en Netherlans, realizado a 40.856 pacientes, donde encontraron el aumento de TFG a lo largo del tiempo en hombres $0,55 \pm 1,47 \mathrm{ml} / \mathrm{min} / 1,73 \mathrm{~m} 2 /$ año, más que las mujeres que fue de $0,33 \pm 1,41 \mathrm{ml} / \mathrm{min} / 1,73 \mathrm{~m} 2 /$ año $(\mathrm{P}<0,001$ para hombres frente a mujeres) $(9,14,15)$.

Con respecto a la edad, los hallazgos del presente estudio guardan estrecha relación con lo encontrado en la literatura mundial, siendo el mayor porcentaje de pacientes con deterioro crónico de la función renal en mayores de 60 años $(75,1 \%)$, esto debido principalmente al deterioro funcional renal por el envejecimiento secundario a la esclerosis glomerular y al conocido deterioro de la TFG de $1 \mathrm{~mL} / \mathrm{min} / 1.73 \mathrm{~m}^{2}$ a partir de los 40 años, como en estudios de Colombia, Perú, Chile, España y China (8),(16)(19).

En cuanto a que la mayoría de la población con ERC proviene de área urbana con un 47\%, que puede deberse a la facilidad de acceso a los servicios de salud de tercer nivel, a diferencia con los pacientes provenientes de municipios más alejados que no cuentan con programas de nefroprotección por lo que los pacientes deben recorrer largas distancias para ser atendidos, siendo similar en otras ciudades de Colombia como Bogotá, que tiene un $87,26 \%$ de pacientes con respecto su departamento, seguida de Medellín y Cali, según la Cuenta de Alto Costo 2019, donde además reporta que el 67,3\% de toda la población con ERC en Colombia se ubica en las ciudades metropolitanas (4).

En relación a los factores clínicos es ampliamente conocido el papel de la HTA en la génesis y el deterioro crónico de la función renal por lo que se ha documentado su mayor prevalencia en este grupo poblacional, como en Colombia 2019 con un $92,43 \%$ de pacientes que tienen algún estadio de ERC, y son hipertensos (4). En el departamento de Nariño la situación no es diferente con un 95\%, al igual que lo encontrado en un estudio realizado en el Hospital General de Medellín en 2015 (20). 
En otros lugares se ha documentado la presencia de la HTA no controlada y su asociación independiente con la progresión de la ERC (16), (19), (21), (22) si bien en el presente estudio no hubo asociación explicativa estadísticamente significativa, probablemente debido a que el $78,5 \%$ de los pacientes hipertensos estaban controlados. Se ha demostrado que el control de la presión arterial es la práctica más estudiada para prevenir el deterioro y las complicaciones de la ERC (15).

En relación a la variable compensación de la TA, esta tuvo un comportamiento protector en el RRc: 0,81 IC95\%0,750,87 con $\mathrm{p}<0,001$, pero al incluirla en el modelo multivariado y ajustando por múltiples variables este valor, aunque no es estadísticamente significativo por atravesar la unidad, se ajusta con un RRa 1,00 IC95\%0,89-1; por lo que se puede deducir que al ajustarlo por otras variables no incluidas en el presente estudio, este puede llegar a comportarse como un factor explicativo asociado a la progresión, justificando la realización de otras investigaciones que exploren estos comportamientos.

Otro factor clínico de gran importancia en la progresión de la ERC es la DM. Se ha explicado ampliamente que este es un factor de riesgo potente para ERC terminal (24). La nefropatía diabética se presenta entre un 25 a un $40 \%$ de los pacientes diabéticos siendo causante de ERC (1), a su vez la proteinuria secundaria a la nefropatía es el principal predictor de progresión de la función renal (25). En Colombia se ha descrito que el $33,17 \%$ de la población tiene DM y ERC en cualquier estadio, datos similares se encontraron en el presente estudio, donde el 25,1\% tuvieron DM. Aunque no se encontró asociación de este factor en la población estudiada (RRc: 1,03 IC95\% 0,97-1,09 p 0,29), no se debe desestimar la información obtenida ya que cuando se analizó la variable HbA1c descompensada, se encontró ajuste de la misma, con la progresión de la ERC, medido por la TFG de $5 \mathrm{~mL} / \mathrm{min} / 1.73 \mathrm{~m}^{2}$ o más. Siendo consecuente con lo reportado en la literatura en donde los niveles elevados de $\mathrm{HbA} 1 \mathrm{C}>7 \%$ (no compensada) es un factor de riesgo para ERC (26), por lo que recomiendan el control de la diabetes como un objetivo prioritario en la disminución del deterioro renal $(6,27,28)$.

En concordancia con diversos estudios a nivel mundial, en el presente trabajo se encontró una prevalencia elevada de HTA y/o DM en pacientes con ERC en cualquiera de sus estadios, con un 98,2\%, y 71,5\%, en Colombia, y en Nariño respectivamente (4), siendo enfermedades causales en todas las fases de la ERC y de la Enfermedad Renal Terminal (2930).

Otro de los factores clínicos asociados al deterioro crónico de la función renal es el exceso de peso, medido en la escala del índice de masa corporal (IMC), dado por sobrepeso y obesidad, en cierta medida como resultado de su aumento en la población en general, pero específicamente en pacientes con ERC genera hiperfiltración glomerular con el consiguiente daño renal, siendo la obesidad un fuerte factor de riesgo para la progresión de la ERC (31), como lo encontrado en un estudio multicéntrico en China, donde la obesidad y la hipertrigliceridemia estaban asociados a la mayor prevalencia de la ERC (19). Estudios que son similares a lo encontrado en Nariño, en donde la obesidad grado I.II-III, si bien no se asociaron significativamente con la progresión de TFG, de
$5 \mathrm{~mL} / \mathrm{min} / 1.73 \mathrm{~m}^{2} \mathrm{o}$ más, se ajustaron con respecto al análisis crudo, esperando en investigaciones futuras poder ahondar en este campo.

Otro hallazgo importante del presente estudio fue el porcentaje un poco más elevado de deterioro de la función renal $(49,2 \%)$, con respecto a lo documentado en un estudio realizado en el Hospital Militar Central de Bogotá en 2014, donde encontraron un deterioro de $\mathrm{TFG}$, en un $40 \%$, siendo además de $5 \mathrm{~mL} /$ $\mathrm{min} / 1.73 \mathrm{~m}^{2}$ o más en el primero y $2 \mathrm{~mL} / \mathrm{min} / 1.73 \mathrm{~m}^{2} \mathrm{o}$ más en el segundo (21).

Los estadios de la ERC clasifican a los pacientes según el grado de deterioro de la función renal, con un menor porcentaje en estadios superiores, como lo reportado en las guías KDIGO 2012, menor de $2 \%$ (1), en Colombia la proporción de ERC en los estadios 3a-b y $4-5$, es de $50,9 \%$ y $11,2 \%$ respectivamente (4), datos similares a los encontrados en el presente estudio en estadios 4-5 con un 9,8\%, pero superados en casi 5 veces lo encontrado a nivel mundial.

Otra variable que nos permite explicar el modelo es el estadio de la ERC, en donde a partir del estadio 3 en adelante, se asoció significativamente con la progresión de la ERC,(E3a-b: RRa:1,41 IC95\% 1,21-1,63; E4-5: RRa:1,62 IC95\%1,36$1,94)$, siendo similar a la literatura donde a partir de los estadios 3a-b, al 5, aumenta la velocidad del deterioro de la función renal (32), debido al nivel creciente de daño glomerular y a la proteinuria subsecuente.

Se encontró que aproximadamente el $50 \%$ de los pacientes del estudio tuvieron un deterioro significativo de la función renal, a pesar de estar en un programa de nefroprotección, esto puede deberse a que se hizo el seguimiento de 2 años, y que no está bien definida la velocidad de deterioro de la función renal en el tiempo, por ser multifactorial, aunque se considera que el periodo mínimo debe ser de 1 año y preferiblemente los seguimientos en esta patología deberían ser de 5 años en adelante, por tratarse de una enfermedad crónica. Otro factor que puede influir en la persistencia de un deterioro importante de la TFG, puede ser debido a que la mayoría de pacientes $88,5 \%$, tienen escolaridad de primaria incompleta o completa, que pueda relacionarse con una menor adherencia al tratamiento complejo y por lo general polimedicado, esto será objeto de estudios posteriores que permitan aclarar estos fenómenos.

Financiación: la investigación fue autofinanciada.

Conflicto de intereses: Los autores declaran que no tienen ningún conflicto de interés. 


\section{Bibliografía.}

1. Eknoyan G, Lameire N, Eckardt K., et al. KDIGO 2012 clinical practice guideline for the evaluation and management of chronic kidney disease. Kidney Int. 2013;3:5-14.

2. Lv, J.-C., Zhang, L.-X. Prevalence and Disease Burden of Chronic Kidney Disease. Renal Fibrosis: Mechanisms and Therapies. 2019;1(1):3-15.

3. Jha V, Garcia-Garcia G, Iseki K, Li Z, Naicker $\mathrm{S}$, Plattner $\mathrm{B}$, et al. Chronic kidney disease: global dimension and perspectives. The Lancet. 2013;382(9888):260-72.

4. Acuña L., Valbuena A., Ramírez P. Situación de la Enfermedad Renal Crónica, la hipertensión arterial y la diabetes mellitus en Colombia 2019. Cuenta de Alto Costo. Fondo Colombiano de Enfermedades de Alto Costo. Bogotá D.C. 2020.

5. Otero A, de Francisco A, Gayoso P, García F; on behalf of the EPIRCE. Study Group. Prevalence of chronic renal disease in Spain: results of the EPIRCE study. Nefrologia 2010;30(1):78-86.

6. Martínez A. Górriz J. Documento de consenso para la detección y manejo de la enfermedad renal crónica. Nefrologia 2014;34(2):243-62.

7. Sellares VL. Enfermedad renal crónica. En: Lorenzo V., Lopez-Goez J., editores. Nefrología al día. Edición del grupo editorial nefrología de la sociedad española de nefrología. España:2010;335-352.

8. Acuña L, Sánchez P, Soler LA, et al. Enfermedad renal crónica en Colombia: prioridad para la gestión de riesgo. Rev. Panam. Salud Pública. 2016;40(1):1622.

9. Halbesma N, Brantsma AH, Bakker SJ., et al. Gender differences in predictors of the decline of renal function in the general population. Kidney Int 2008.

10. Coordinación metodológica de la Cuenta de Alto Costo. Contenidos mínimos indispensables para la gestión del riesgo renal en un programa de atención a pacientes adultos con enfermedad renal crónica, sin terapia de reemplazo renal en Colombia. Definiciones técnicas basadas en evidencia [Internet]. 2011. Disponible en:https://www.minsalud.gov.co/sites/ $\mathrm{rid} /$ Lists/BibliotecaDigital/RIDE/INEC/CAC/ programas_ERC.pdf

11. Fondo Colombiano de Enfermedades de Alto Costo. Nefroprotección: Estrategia fundamental para evitar, controlar y retrasar el daño renal. Boletín de Información Técnica especializada. 10 de marzo de 2016;2(2).
12. Espinal L., Factores asociados a Enfermedad renal crónica en estadios pre diálisis [Trabajo de grado]. Medellín (Antioquia): Universidad CES;2015.

13. Bertakis KD, Azari R, Helms LJ., et al. Gender differences in the utilization of health care services. J Fam Pract. 2000 Feb;49(2):147-52.

14. Bardají A, Martínez-Vea A. Enfermedad renal crónica y corazón. Un continuo evolutivo. Rev Esp Cardiol. 2008;61(Supl.2):41-51.

15. Vikse BE, Irgens LM, Leivestad T, et al. Low birth weight increases risk for endstage renal disease. Journal of the American Society of Nephrology: JASN 2008;19(1):151-157.

16. Bravo J.,Chavez R., Galvez J., et al. Progresión de la Enfermedad renal crónica en un hospital de referencia de la seguridad social de Perú 2012-2015. Rev Perú Med Exp Salud Publica 2017;34(2):209-17.

17. Zúñiga SM C, Müller O H, Flores O M. Prevalencia de enfermedad renal

crónica en centros urbanos de atención primaria. Rev Médica Chile 2011;139:1176-84.

18. Martínez-Castelao A, Górriz JL, Bover J, Segura-de la Morena J, Cebollada J, Escalada J, et al. [Consensus document for the detection and management of chronic kidney disease]. Endocrinol Nutr Organo Soc Esp Endocrinol Nutr. 2014 Nov;61(9):e25-43.

19. Andong Ji., Chunlei Pan., Hongxia W., et al. Prevalence and Associated Risk Factors of Chronic Kidney Disease in an Elderly Population from Eastern China. International Journal of Environmental Research and Public Health 2019;16(22):4383.

20. Espinal L., Factores asociados a Enfermedad renal crónica en estadios pre diálisis [Trabajo de grado]. Medellín (Antioquia): Universidad CES;2015.

21. Camargo JA., Vargas JG., DAchiadi R., et al. Factores de riesgo para la progresión de la enfermedad renal crónica en pacientes con nefropatía diabética estadios 3-4 del Servicio de Nefrología del Hospital Militar Central de Bogotá. Rev. Nefrología 2014;1(1).

22. Zheng Y, Tang L, Zhang W., et al. Applying the new intensive blood pressure categories to a nondialysis chronic kidney disease population: the Prevalence, Awareness and Treatment Rates in Chronic Kidney Disease Patients with Hypertension in China survey. Nephrol Dial Transplant. 2020 Jan 1;35(1):155-161.

23. Vikse BE, Irgens LM, Leivestad T, et al. Low birth weight increases risk for endstage renal disease. Journal of the American Society of Nephrology: JASN 2008;19(1):151-157. 
24. Lorenzo V, SanchezE, Vega N, et al. Renal replacement therapy in the Canary Islands: demographic and survival analysis. J Nephrol 2006;19(1):97-103.

25. Lorenzo V, Saracho R, Zamora J, Rufino M, Torres A: Similar renal decline in diabetic and nondiabetic patients with comparable levels of albuminuria. Nephrol Dial Transplant 2010, 25(3):835-841.

26. Hsu CY, Bates DW, Kuperman GJ, Curhan GC: Diabetes, hemoglobin $\mathrm{A}(1 \mathrm{c})$, cholesterol, and the risk of moderate chronic renal insufficiency in an ambulatory population. Am J Kidney Dis 2000, 36(2):272-281.

27. European Dialysis and Transplant Association European Renal Association. Guideline development: Clinical Practice Guideline on management of patients with diabetes and chronic kidney disease stage $3 b$ or higher. Nephrology, dialysis, transplantation, 2015;30Suppl 2:1-142.

28. Gomez-Huelgas R., Martinez- Castelao A., Documento de Consenso sobre el tratamiento de la diabetes tipo 2 en el paciente con enfermedad renal crónica. Nefrología 2013;1(34):0-138.

29. Dearbhla K., Rothwell PM. Disentangling the multiple links between renal dysfunction and cerebrovascular disease. Neurol Neurosurg Psychiatry. 2020 Jan;91(1):88-97.

30. Gorostidi M, de la Sierra A, Gonzalez-Albarran O, et al. Abnormalities in ambulatory blood pressure monitoring in hypertensive patients with diabetes. Hypertens. Res. 2011; 34(11):1185-1189.

31. Kramer H, Luke A, Bidani A., et al. Obesity and prevalent and incident CKD: the Hypertension Detection and Follow-Up Program. Am J Kidney Dis 2005;46(4):587-594.

32. Provenzano M., Chiodini P., Minutolo R. Reclassification of chronic kidney disease patients for end-stage renal disease risk by proteinuria indexed to estimated glomerular filtration rate: multicentre prospective study in nephrology clinics. Nephrol. Dial Transplant. 2020;1;35(1):138-147. 